\title{
A Case of Adenoid Cystic Carcinoma of the Parotid Gland
}

\author{
-An Immunohistological Study- \\ Yasuo TAKAYAMA ${ }^{1}$, Tsubura SUZUKI ${ }^{1}$, Takashi SHIRANO ${ }^{1}$, \\ Hiromitsu YAMANOI ${ }^{1}$, Minoru HORI ${ }^{1}$, Hiroshi TANAKA ${ }^{1}$, \\ Kaoru KUSAMA ${ }^{2}$ and Itaru MORO ${ }^{2}$
}

(Received 15 February and accepted 27 March 1990)

Key words: adenoid cystic carcinoma, parotid gland, immunohistochemistry

\begin{abstract}
We treated a case of adenoid cystic carcinoma that occurred in the right parotid gland of a 51-year-old woman. Although the tumor, including part of the parotid gland, was resected based on a clinical diagnosis of pleomorphic adenoma, it was later diagnosed as adenoid cystic carcinoma of mixed cribriform-solid type by histopathological examination after resection. Therefore, postoperative irradiation with a dose of $51 \mathrm{~Gy}$ was performed.

Immunohistological examination of the resected specimen showed that the profiles of carbohydrate antigens were Lewis $\left(\mathrm{a}^{-}, \mathrm{b}^{+}\right)$in both the tumor and surrounding normal parotid tissue. NCC-SG-007 antigen was observed in the tumor tissue but not in the normal parotid gland, suggesting that this antigen was associated with the tumor.
\end{abstract}

\section{Introduction}

Adenoid cystic carcinoma is a malignant tumor of the salivary gland frequently occurring in the submandibular gland among the major salivary glands and in the palatine gland among the minor salivary glands, whereas its incidence in the parotid gland is relatively low ${ }^{[1-3]}$.

We report here a rare case of this tumor that occurred in the right parotid gland of a 51-year-old woman, and which was examined immunohistologically. In addition, to examine the expression of Lewis blood group substances, related antigens and NCC-SG-007 antigen in the tumor, immunohistochemistry was performed.

\footnotetext{
高山泰男, 鈴木 円, 白野隆史, 山野井弘充, 堀 稔, 田中 博, 草間 薰, 茂呂 周：

1 1st Department of Oral Surgery, Nihon University School of Dentistry

2 Department of Pathology, Nihon University School of Dentistry

To whom all correspondence should be addressed: Dr. Hiroshi TANAKA, 1st Department of Oral Surgery, Nihon University School of Dentistry, 1-8-13 Kanda-Surugadai, Chiyoda-Ku, Tokyo 101, JAPAN.
} 


\section{Case Report}

Patient: 51-year-old woman.

Initial examination: May 26, 1988.

Chief complaint: A mass in the posterior region of the right mandibular ramus.

Past and family histories: Non-contributory.

Clinical course of the present disease: The patient first noticed a mass in the posterior portion of the right mandibular ramus six months previously, but did not seek treatment because of the absence of pain. When this patient visited a dentist for treatment of dental caries, she was recommended by the dentist to undergo an examination of the mass and was referred to our department.

Present condition: A slight swelling was noted in the posterior portion of the mandibular ramus in the vicinity of the angle of the right mandible, and a mass was palpated under the skin in this region (Fig. 1). It was elastic and hard, and of walnut size with a clear border and smooth surface. The mass showed no strong adhesion to the surrounding tissues, and there was no abnormal appearance of the skin in this region. The patient had no trismus, spontaneous pain, tenderness or facial nerve symptoms such as paralytic lagophthalmus, or inability to whistle. There were neither abnormal findings in the oral cavity nor noticeable changes in the lateral wall of the oropharynx.

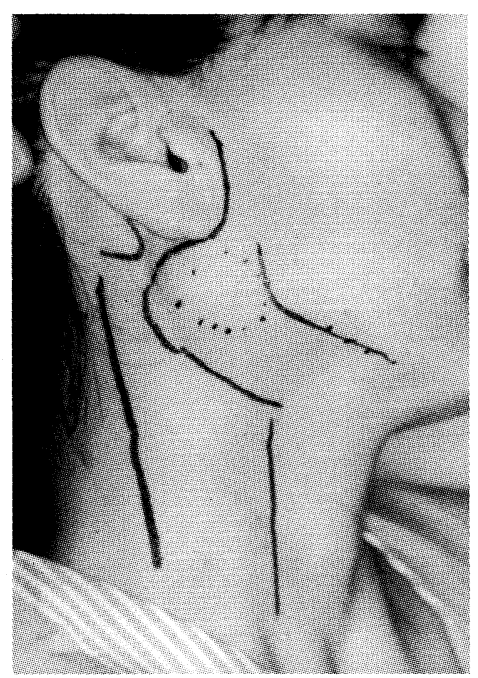

Fig. 1 Facial appearance

Regional lymph nodes: Two right submandibular lymph nodes were palpable. One was the size of the little finger tip and the other was of soybean size. Both were mobile and non-tender. No cervical lymphadenopathy was evident.

Roentgenographic findings: An orthopantomogram and chest X-ray film revealed no abnormal images. 


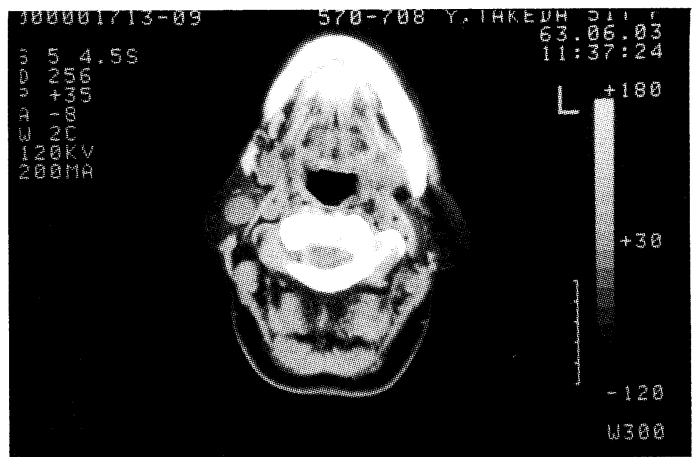

Fig. 2 CT findings: spherical mass with uniform density at the posterior portion of the right mandibular ramus

CT findings: A spherical mass of uniform density was observed in the posterior portion of the right mandibular ramus (Fig. 2).

$R I$ scintigram findings: In ${ }^{67} \mathrm{Ga}$ and ${ }^{99 \mathrm{~m}} \mathrm{Tc}-\mathrm{MDP}$ scintigrams, no abnormal uptake was seen in the right parotid region or any other regions of the body.

Laboratory data showed no abnormal values.

Treatment and clinical course: Based on a clinical diagnosis of pleomorphic adenoma of the right parotid gland, the tumor was resected using the following procedure: an S-shaped skin incision was made from the preauricular to the submandibular region; the main trunk of the facial nerve was exposed, and its marginal mandibular branch was identified and traced to the periphery. The tumor was then removed including part of the parotid gland.

On histopathological examination of the resected specimen, the tumor was diagnosed as adenoid cystic carcinoma. Therefore, postoperative irradiation therapy with a betatron usig a dose of 51 Gy to the right parotid region was started 2 months after surgery. At the time of writing, 17 months after the operation, there are no complications such as facial nerve paralysis or Frey's syndrome, signs of local recurrence or distant metastasis.

Gross findings of the resected specimen: The resected mass measured $23 \times 16$ $\mathrm{mm}$ and was spheroid in shape. The cut surface was yellowish-gray and a relatively

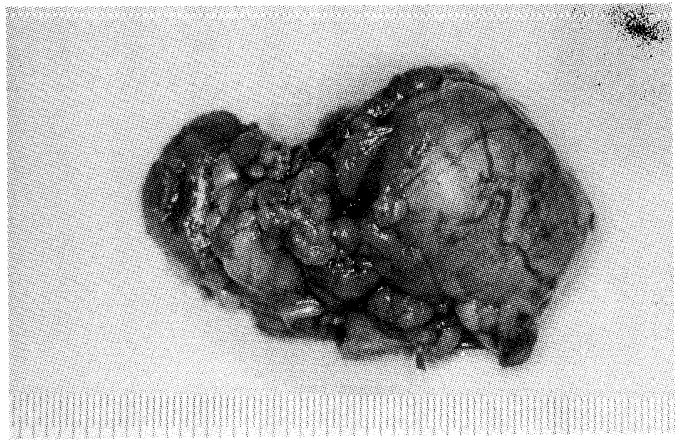

Fig. 3 Gross appearance of the resected specimen 


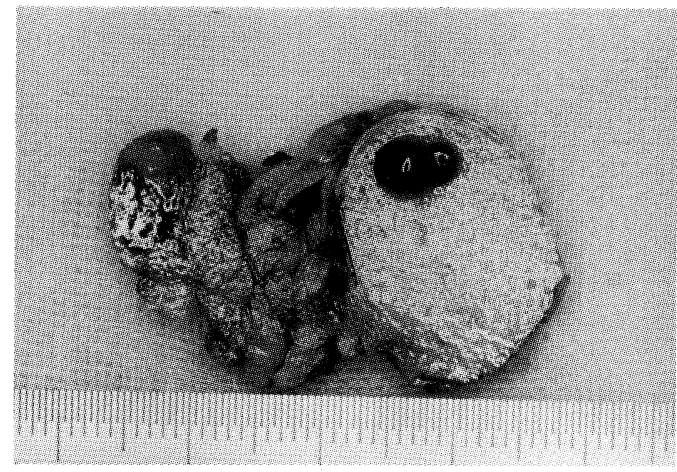

Fig. 4 Cut surface of sepecimen. Cystic cavity is formed inside the tumor mass.

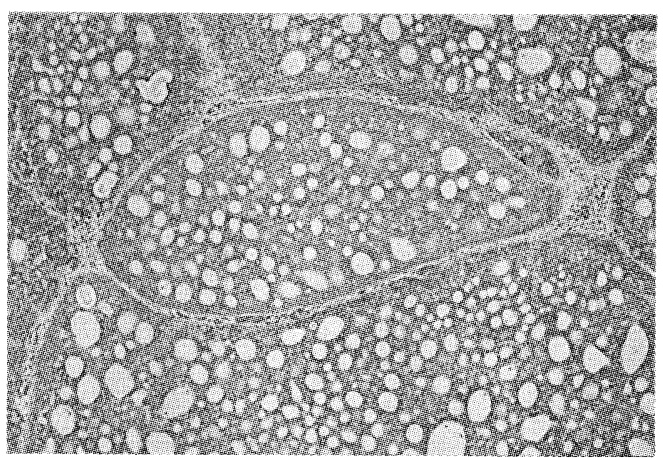

Fig. 5 Cribriform nests of tumor cells

large cystic cavity was present inside the mass (Figs. 3, 4).

Histopathological findings: The resected mass was composed of tumorous tissue surrounded by a fibrous capsule. The parenchyma consisted of relatively small cuboidal or polygonal cells with spheroid nuclei, and formed a cribriform or a solid follicular pattern. In the cribriform nests, hyalinoid and mucinous substances were present within the cavities (Fig. 5). Interstitium was composed of fibrous connective tissues, and congestive regions sparsely surrounded the tumor nests, and mild cell infiltration consisting mainly of plasma cells was observed. Tumor cells infiltrated into, but not beyond the capsule.

The histopathological diagnosis was adenoid cystic carcinoma.

Immunohistological findings: Localization of Lewis a $\left(\mathrm{Le}^{\mathrm{a}}\right)$, Lewis $\mathrm{b}\left(\mathrm{Le}^{\mathrm{b}}\right)$, CA 19-9 and CA 50 antigen was determined immunohistologically using corresponding antibodies by the avidin-biotin-peroxidase complex (ABC) method ${ }^{[4]}$. In addition, the monoclonal antibody NCC-SG-007 was used to detect carbohydrate antigens lacking terminal sialic acid, since this antibody was originally developed using adenoid cystic carcinoma as the antigen ${ }^{[5]}$. 


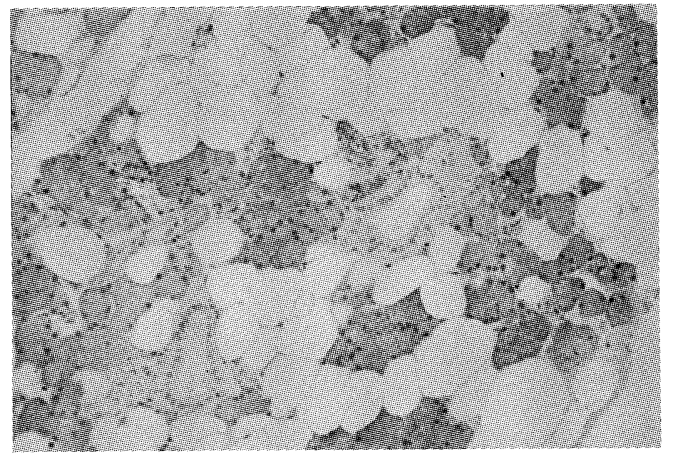

Fig. 6 Localization of $\mathrm{Le}^{\mathrm{b}}$ antigen of normal parotid gland tissues

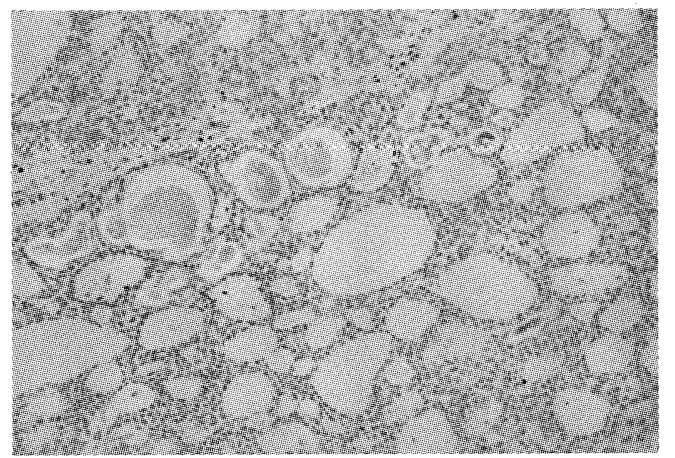

Fig. 7 Localization of NCC-SG-007 antigen of tumor tissues

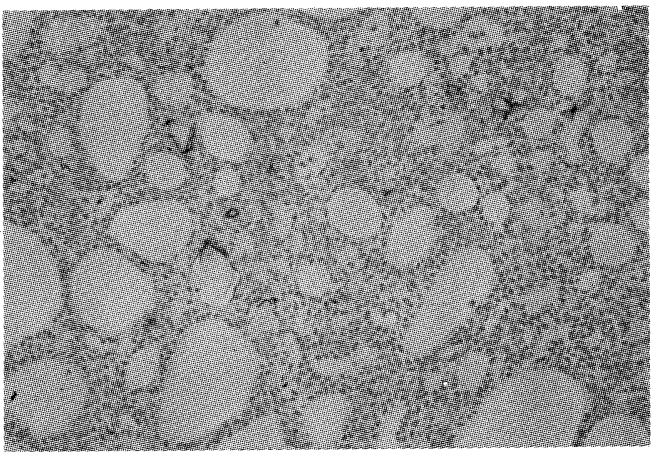

Fig. 8 Localization $\mathrm{Le}^{\mathrm{b}}$ antigen of tumor tissues 
In normal parotid gland tissue adjacent to the tumor, there was no localization of Le ${ }^{\mathrm{a}}$, CA 19-9, CA 50 or NCC-SG-007 antigen, whereas Le ${ }^{\mathrm{b}}$ was seen in the epithelium and contents of intercalated ducts and some acinic cells (Fig. 6).

In the tumor tissues, $\mathrm{Le}^{\mathrm{a}}$, CA $19-9$ or $\mathrm{CA} 50$ was not detected, but the NCC-SG-007 antigen was observed in the contents of duct-like structures (Fig. 7). $\mathrm{Le}^{\mathrm{b}}$ was localized on the free surfaces and in the contents of duct-like structures as well as on tumor cells (Fig. 8).

\section{Discussion}

Adenoid cystic carcinoma frequently occurs in the submandibular gland ${ }^{[6]}$ but rarely in the parotid gland. It has been reported that this tumor accounts for only about $2 \%$ of all tumors of the parotid gland ${ }^{[1,2]}$. Although some investigators consider that adenoid cystic carcinoma grows slowly and rarely metastasizes to regional lymph nodes, it has been commonly reported that this tumor shows marked infiltration into surrounding tissues, often forming local recurrence and distant metastasis ${ }^{[7,8]}$. Moreover, it has been described that the tumor often infiltrates into the nerve sheath, resulting in pain at an early stage and facial paralysis in cases affecting the parotid gland ${ }^{[8,9]}$. Our present patient noticed a mass that developed in a period as short as about six months, but had no spontaneous pain, tenderness or facial nerve symptoms.

Histopathologically, adenoid cystic carcinoma is generally classified into three types based on parenchymal structure ${ }^{[10,11]}$, i.e. a cribriform, tubular or solid pattern. Perzin et al. ${ }^{[10]}$ pointed out that such histological types were related to the degree of malignancy and prognosis of the tumor, and that the solid type was the most malignant with a poor prognosis, followed by the cribriform type and tubular type in that order. As for the present case, the tumor appeared to be a mixture of the cribriform and solid types, and was therefore thought to have

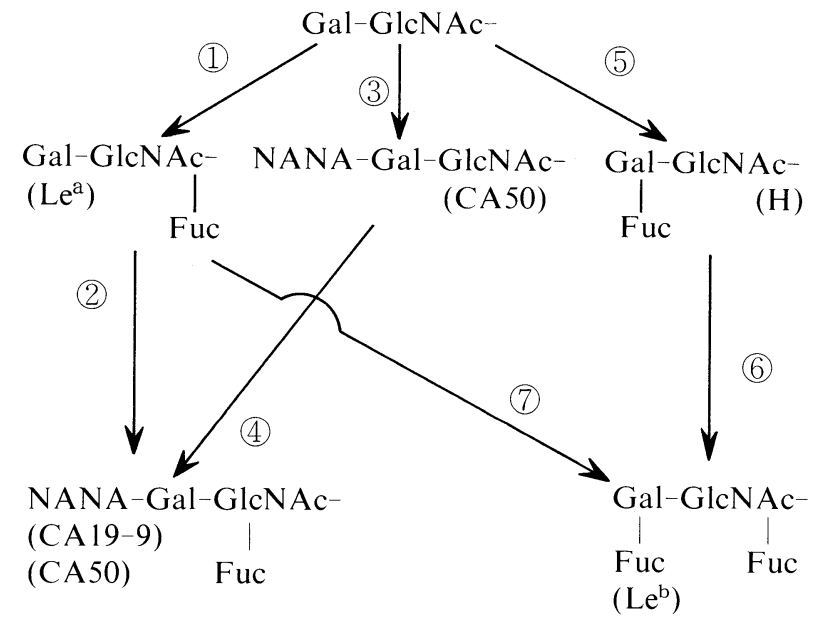

Fig. 9 Schematic pathway of carbohydrate synthesis 
relatively high malignancy from a histological viewpoint.

Immunohistologically, the following results were obtained: (1) As to Lewis type, the normal parotid tissue in the vicinity of the tumor was Le $\left(a^{-}, b^{+}\right)$. (2) Although the Lewis type of the tumor tissue was similar to that of the normal parotid tissue, carbohydrate antigen NCC-SG-007, which was not seen in the normal parotid tissue, was localized in the tumor. (3) No Le ${ }^{a}$, CA 19-9 or CA 50 was observed. From these results, it was assumed that pathways 1, 2, 3, 4 and 7 shown in Fig. 9 were absent in this case, whereas pathways 5 and 6 were involved in carbohydrate synthesis in both normal and tumor tissues.

Various techniques, including sialography, CT scan, RI scintigraphy and ultrasonography, have been utilized for preoperative diagnosis of adenoid cystic carcinoma. However, it is not very easy to differentiate adenoid cystic carcinoma from benign tumors such as pleomorphic adenoma because of its relatively slow growth, chronic clinical course, tumor size and encapsulation by fibrous connective tissue. If malignant tumor cannot be ruled out preoperatively, it is common to undertake histological examination of a biopsy specimen. However, open biopsy is usually thought to be undesirable in cases of salivary gland tumor because it has been reported that local infiltration and lung metastasis may occur easily ${ }^{[12,13]}$. As for our present case, the tumor was removed along with part of the parotid gland based on an initial clinical diagnosis of pleomorphic adenoma; however, the tumor was diagnosed postoperatively as adenoid cystic carcinoma based on its histopathology.

As for the treatment of adenoid cystic carcinoma, radical surgery with wide resection is considered to be the first-choice treatment, as this tumor is insensitive to irradiation or chemotherapy. MURAKAMI et al. ${ }^{[14]}$ have stated that superficial lobectomy is sufficient treatment for parotid gland tumors, even those of a malignant nature, except for cases with marked infiltration into tissues around the facial nerve and into the deep lobe of the parotid gland. In the present case, we performed superficial lobectomy and considered that a reasonable safety margin had been attained because the tumor was found to be encapsulated with fibrous connective tissue and had not infiltrated beyond the capsule on histopathological examination. In surgery for salivary gland tumors with no definite clinical diagnosis, the necessity of diagnosis by frozen section during surgery is emphasized.

When the postoperative diagnosis is different from the preoperative diagnosis, supplementary surgery, chemotherapy and/or irradiation may be performed. Although adenoid cystic carcinoma is thought to be insensitive to irradiation, some investigators recommend postoperative irradiation therapy, even if sufficiently wide resection has been performed ${ }^{[15]}$. BARDwIL et al. ${ }^{[16]}$ recognized the ineffectiveness of irradiation, but stated that this therapy should be reserved for inoperable recurrent cases, incompletely resected tumors and those suspected to have an unsafe surgical margin. Therefore, postoperative treatment with betatron irradiation at $51 \mathrm{~Gy}$ was applied to our patient even though no tumor infiltration beyond the capsule was observed.

Seventeen months after surgery, our patient shows no evidence of recurrence or metastasis. 


\section{References}

[1] SPIro, R. H., Huvos, A. G. and STrong, E. W.: Adenoid cystic carcinoma of salivary origin: a clinicopathologic study of 242 cases, Am. J. Surg., 128, 512-520, 1974

[2] Eneroth, C. M.: Salivary gland tumors in the parotid gland, submandibular gland, and the palate region, Cancer, 27, 1415-1418, 1971

[3] Mukai, H., Gotanda, M., Hwang, B. T., Tomori, Y., Sugihara, K. and Yamashita, S.: Two cases of adenoid cystic carcinoma, Jpn. J. Oral. Maxillofac. Surg., 29, 564-569, 1983 (in Japanese)

[4] Hsu, S. M., RAINe, L. and FAnger, H.: Use of avidin-biotin-peroxidase complex (ABC) in immunoperoxidase techniques: a comparison between ABC and unlabeled antibody (PAP) procedures, J. Histochem. Cytochem., 29, 577-580, 1981

[5] Kusama, K., Sekine, T., Adachi, M., Ogaki, H., Hirota, T., Morinaga, S., Hasegawa, H., Hayakawa, N., Moro, I. and S Sto, S.: Derivation of a murine monoclonal antibody useful for immunohistochemical diagnosis of human adenoid cystic carcinoma and stomach cancer, Jpn. J. Cancer Res. (Gann), 78, 952-959, 1987

[6] Simons, J. N., Beahrs, O. H. and Woolner, L. B.: Tumors of the submaxillary gland, Am. J. Surg., 108, 485-494, 1964

[7] Kawamura, G.: Malignant tumor. In Current Oral Surgery, 2nd ed., 905, Nakamura, H., Unno, T. and Iroh, H., Eds., Ishiyaku Publisher Co. Ltd., Tokyo, Japan, 1981 (in Japanese)

[8] Ishikawa, G.: Oral pathology Vol. 2, 749-753, Nagasue Books, Kyoto, Japan, 1982 (in Japanese)

[9] Noguchi, T., Sekitani, T., Kanesada, K., Matsuo, T., Imate, Y. and Yamashita, H.: Adenoid cystic carcinoma: A clinicopathologic study of 12 cases, J. Otolaryngol. Jpn., 88, 776-783, 1985

[10] Perzin, K. H., Gullane, P. and Clairmont, A. C.: Adenoid cystic carcinomas arising in salivary glands: A correlation of histologic features and clinical course, Cancer, 42 , 265-282, 1978

[11] Senba, O., Satoh, T., Miyahara, H., Yoshino, K., Umatani, K. and Ishiguro, S.: Clinical and histological studies of adenoid cystic carcinoma, Jap. J. Cancer Clin., 29, 287-291, 1983 (in Japanese)

[12] Mason, D. K. and Chisholm, D. M.: Surgical Treatment of Salivary Gland Disease. Salivary Glands in Health and Disease, 232-241, W. B. Saunders Co. Ltd., London, England, 1975

[13] Murakami, Y.: Preoperative Diagnosis of Parotid Gland Tumor. Clinical Management of Parotid Gland Diseases, 89-93, OKudA, M., Ed., Medical Education Publishers, Tokyo, Japan, 1984 (in Japanese)

[14] Murakami, Y., Ino, T., Horiuchi, M., Inokari, T., Ootsuki, J., Satoh, M., Ootsuka, M., Nameki, H. and Nishida, K.: Parotid tumors, a report of seventy cases, Otolaryngology, 48, 589-602, 1976 (in Japanese)

[15] Horiuchi, J., Shibuya, H., Takeda, M. and Suzuki, M.: Results of radiation therapy for carcinoma of the salivary gland, Jap. J. Cancer Clin., 32, 117-122, 1986 (in Japanese)

[16] Bardwil, J. M., Reynolds, C. T., I Ianez, M. L. and Luna, M. A.: Report of one hundred tumors of the minor salivary glands, Am. J. Surg., 112, 493-497, 1966 